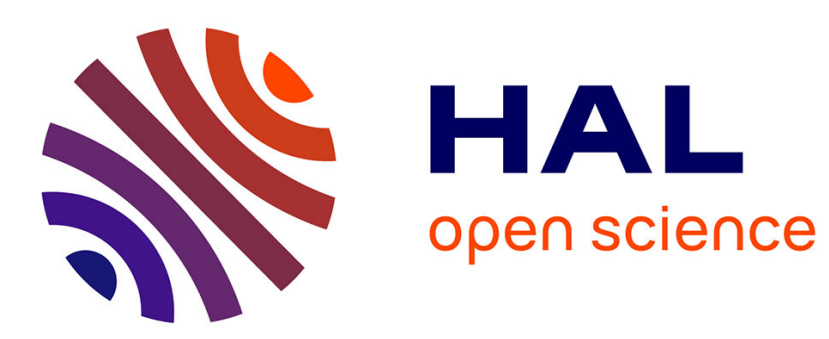

\title{
Homogenization of Random Parabolic Operator with Large Potential
}

\author{
Fabien Campillo, Marina Kleptsyna, Andrey Piatnitski
}

\section{To cite this version:}

Fabien Campillo, Marina Kleptsyna, Andrey Piatnitski. Homogenization of Random Parabolic Operator with Large Potential. RR-3520, INRIA. 1998. inria-00073164

\section{HAL Id: inria-00073164 https://hal.inria.fr/inria-00073164}

Submitted on 24 May 2006

HAL is a multi-disciplinary open access archive for the deposit and dissemination of scientific research documents, whether they are published or not. The documents may come from teaching and research institutions in France or abroad, or from public or private research centers.
L'archive ouverte pluridisciplinaire HAL, est destinée au dépôt et à la diffusion de documents scientifiques de niveau recherche, publiés ou non, émanant des établissements d'enseignement et de recherche français ou étrangers, des laboratoires publics ou privés. 


\section{Homogenization of random parabolic operator with large potential}

Fabien Campillo — Marina Kleptsyna — Andrey Piatnitski

$$
\mathbf{N}^{\circ} 3520
$$

Octobre 1998

THÈME 4 



\title{
Homogenization of random parabolic operator with large potential
}

\author{
Fabien Campillo*, Marina Kleptsyna ${ }^{\dagger}$, Andrey Piatnitski ${ }^{\ddagger}$ \\ Thème 4 - Simulation et optimisation \\ de systèmes complexes \\ Projet Sysdys
}

Rapport de recherche $\mathrm{n}^{\circ} 3520$ - Octobre 1998 - 32 pages

\begin{abstract}
We study the averaging problem for a divergence form random parabolic operators with a large potential and with coefficients rapidly oscillating both in space and time variables. We assume that the medium possesses the periodic microscopic structure while the dynamics of the system is random and, moreover, diffusive.
\end{abstract}

Key-words: Random operators, homogenization, averaging

This work was supported by the CNRS Program "Modélisation et simulation numérique", it has partially been done during the stay in Marseille of Marina Kleptsyna (from October to December 1997 supported by InRIA Sophia Antipolis and the "Communauté des Villes de Marseilles") and Andrei Piatnitski (December 1997, supported by the Université de Provence).

* SYSDYS Project, INRIA/LATP, IMT, 38 rue F. Joliot-Curie, 13451 Marseille cedex, France - campillo@sophia.inria.fr

$\dagger$ Moscow State University of Communications, Obraztsova, 15, Moscow 101475 Russia - asarin@ippi.ac.msk.su

$\ddagger$ Lebedev Physical Institute, Leninski prospect 53, Moscow 117924 Russia andrey@sci.lpi.msk.su. 


\section{Homogénéisation d'opérateurs paraboliques aléatoires avec grand potentiel}

Résumé : On étudie le problème de moyennisation pour des opérateurs paraboliques aléatoires sous forme divergence dans le cas d'un grand potentiel et avec des coefficients rapidement oscillant en temps aussi bien qu'en espace. On suppose que le milieu possède une structure microscopique périodique alors que la dynamique du système est aléatoire et de plus diffusive.

Mots-clés : Opérateurs aléatoires, homogénéisation, moyennisation 


\section{Contents}

1 Introduction 4

$\begin{array}{lll}2 & \text { The setup } & 7\end{array}$

2.1 Notations ...................... 7

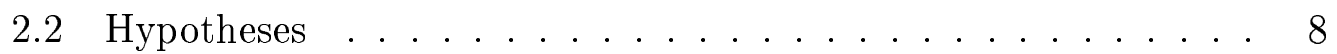

2.3 Auxiliary results .................... 10

3 Main results $\quad 12$

3.1 Case $\alpha<2 \quad \ldots \ldots \ldots \ldots$. . . . . . . . . . . . . . . 12

3.2 Case $\alpha=2 \ldots \ldots \ldots \ldots \ldots$

3.3 Case $\alpha>2$. . . . . . . . . . . . . . . . . . . 14

3.4 Comments ........................ 15

4 Proof of the main results $\quad 18$

4.1 Decomposition of the solution $u^{\varepsilon}$ - Auxiliary homogenization problem ...................... 18

4.2 Tightness result for auxiliary homogenization problem . . . . . . 18

4.3 Description of homogenized operator for auxiliary problem Convergence in probability . . . . . . . . . . . 20

4.4 Convergence in law of the solution of initial problem - Description of the limit distribution . . . . . . . . . . . 28

$\begin{array}{ll}\text { References } & 31\end{array}$

$\mathrm{RR} \mathrm{n}^{\circ} 3520$ 


\section{Introduction}

We study the averaging problem for a divergence form random parabolic operators with a large potential and with coefficients rapidly oscillating both in space and time variables.

The homogenization problems for various random structures are widely discussed in the physical and mathematical literature, see, for example, Jikov et al [7] and its bibliography.

The first results for the divergence form random elliptic operators with stochastically homogeneous coefficients were obtained by Kozlov [9]. Then, this method was formalized and generalized by Papanicoulaou-Varadhan [12]. Later, many other random structures were investigated, among them the random porous media Hornung [6], the equations with lower order terms AvellanedaMajda [1], Fannjiang-Papanicolaou [5]. This led to the appearance of new methods such as Bourgeat et al.[4], Olla [11].

Currently, there are several mathematical approaches which allow to examine homogenization problem in random media, but in all the studied models the randomness in spatial variables and the presence of a group of transformation preserving some probability measure, are supposed.

In our model we assume that the medium possesses the periodic microscopic structure while the dynamics of the system is random and, moreover, diffusive. The equations without potential were previously considered in KleptsynaPiatnitski [8].

The problems of this type appear, for instance, when one studies the macroscopic behavior of micro-inhomogeneous locally periodic dissipative media whose properties change randomly in time, or the effect of random action on locally periodic structures with large dissipation.

The corresponding parabolic operator is of the form:

$$
u_{t}(t, x)-\operatorname{div}\left[a\left(\frac{x}{\varepsilon}, \xi_{t / \varepsilon^{\alpha}}\right) \nabla u(t, x)\right]-\varepsilon^{-\beta} c\left(\frac{x}{\varepsilon}, \xi_{t / \varepsilon^{\alpha}}\right) u(t, x), \quad x \in \mathbb{R}^{n},
$$

where $\varepsilon$ is a small positive parameter, $\alpha, \beta>0$, the coefficients $a^{i j}(z, y)$ and $c(z, y)$ are periodic in the first argument, and $\xi_{t}$ is a diffusion process with values in $\mathbb{R}^{d}$ solving the following stochastic differential equation:

$$
d \xi_{t}=B\left(\xi_{t}\right) d t+\sigma\left(\xi_{t}\right) d W_{t} .
$$


The parameter $\alpha$ represents the ratio between space and time microscopic length scales; in the "self-similar" case $\alpha=2$, a coupling between space and time averaging appears.

Regarding the process $\xi_{s}$, we suppose good mixing and localization properties. Our approach requires a sufficiently fast decay of the density of the invariant measure of $\xi_{s}$ at the infinity; for instance, a condition of the Khasminski type $B(y) \cdot y /|y|<-c, c>0$, is sufficient. In fact, quoting a new work Pardoux-Veretennikov [13], we will assume a weaker condition:

$$
B(y) \cdot \frac{y}{|y|}<-c|y|^{\mu}
$$

for some $\mu>-1$ and $c>0$. The exact assumptions on the regularity of coefficients and the process $\xi$ are given in Section 2.2.

The relation between $\alpha$ and $\beta$ is of great importance. If $\beta<\alpha / 2 \wedge 1$ then the presence of the potential $c(z, y)$ is neglectable in a proper sense. If, on the contrary, $\beta>\alpha / 2 \wedge 1$ then, in general, the family of solutions is not compact. The only exception is the case where $\alpha>2$ and where the mean value of $c(z, y)$ in $z$ is equal to zero for all $y$. In this case, the proper choice is $\beta=\alpha / 2$ instead of $\beta=1$.

We will show that for $\beta=\alpha / 2 \wedge 1$ under the natural regularity assumptions, the averaging results hold while the structure of the limit problem depends crucially on whether $\alpha=2$, or $\alpha<2$ or $\alpha>2$.

If $\alpha>2$ then the family of solutions of corresponding Cauchy problems converges in probability in a proper functional space to the solution of the Cauchy problem for parabolic operator with constant nonrandom coefficients. This result looks like classical homogenization result with the only difference that we obtain convergence in probability. The almost sure convergence is an open question.

If $\alpha \leq 2$ then the family of measures generated by the solutions, converges weakly to the unique solution of the limit martingale problem which involves the one-dimensional Brownian motion. The formula for the coefficients of the limit problem are rather different in the cases $\alpha=2$ and $\alpha<2$.

These results show that the presence of stochastic dynamics might change essentially the limit behavior of solutions. It is interesting to note that in a particular case $c(z, y) \equiv 0$ the limit equation is always deterministic KleptsynaPiatnitski [8].

$\mathrm{RR} \mathrm{n}^{\circ} 3520$ 
In Section 2 the precise setting of the problem is given and some auxiliary statements are quoted.

The main results of the paper are formulated in Section 3.

The next section is devoted to the proof of the main statements. It should be noted that, in general, the expectation of the norm of $u^{\varepsilon}$ does not admit uniform in $\varepsilon$ upper bound. Thus, we cannot apply the standard technique in order to obtain weak compactness results. Instead, we decompose $u^{\varepsilon}$ into the product:

$$
u^{\varepsilon}(t, x)=\exp \left(\frac{1}{\varepsilon^{1 \wedge \frac{\alpha}{2}}} \int_{0}^{t}\left\langle c\left(\cdot, \xi_{s / \varepsilon^{\alpha}}\right)\right\rangle d s\right) v^{\varepsilon}(t, x)
$$

and introduce in this way new unknown function $v^{\varepsilon} ;\langle\cdot\rangle$ stands for the mean value of a periodic function.

For the family of functions $v^{\varepsilon}$ we obtain a priori estimates, prove the convergence of $v^{\varepsilon}$ in probability to a deterministic limit in a suitable functional space and find the auxiliary homogenized equation for the limit function. To this end we introduce a family of correctors involving the solutions of proper Poisson equations and vanishing in a suitable sense as $\varepsilon \rightarrow 0$, and, with the help of Itô's calculus, reduce the problem to studying the limiting behavior of a family of semi-martingales. Then, the technique developed in Viot [14], Bouc-Pardoux [3], can be applied.

The description of the limit distribution of $\exp \left(\frac{1}{\varepsilon^{1 \wedge \frac{\alpha}{2}}} \int_{0}^{t}\left\langle c\left(\cdot, \xi_{s / \varepsilon^{\alpha}}\right)\right\rangle d s\right)$ is due to [13], where the weak convergence of $\frac{1}{\varepsilon^{\alpha / 2}} \int_{0}^{t}\left\langle c\left(\cdot, \xi_{s / \varepsilon^{\alpha}}\right)\right\rangle d s$ has been proved.

Finally, the passage to the limit in the product relies on the deterministic nature of the limit of $v^{\varepsilon}$. 


\section{The setup}

We consider the asymptotic behavior of the solution of the following Cauchy problem as $\varepsilon \downarrow 0$ :

$$
\begin{aligned}
& u_{t}^{\varepsilon}(t, x)=\operatorname{div}\left[a\left(\frac{x}{\varepsilon}, \xi_{\frac{t}{\varepsilon \alpha}}\right) \nabla u^{\varepsilon}(t, x)\right]+\frac{1}{\varepsilon^{1 \wedge} \frac{\alpha}{2}} c\left(\frac{x}{\varepsilon}, \xi_{\frac{t}{\varepsilon^{\alpha}}}\right) u^{\varepsilon}(t, x), \\
& u^{\varepsilon}(0, x)=u_{0}(x), x \in \mathbb{R}^{n}, t \in[0, T]
\end{aligned}
$$

where $\alpha>0$ is a parameter and $T>0$ is fixed.

The coefficients $a(z, y)$ and $c(z, y)$ are periodic in $z$ (or $z$ belongs to the unit torus $\mathbb{T}^{n}=\mathbb{R}^{n} / \mathbb{Z}^{n}$ ), and $\left\{\xi_{t}\right\}_{t \geq 0}$ is stationary ergodic diffusion process, with values in $\mathbb{R}^{d}$, given by

$$
d \xi_{t}=B\left(\xi_{t}\right) d t+\sigma\left(\xi_{t}\right) d W_{t}
$$

Let us introduce the following operators:

- the infinitesimal generator of the diffusion process $\left\{\xi_{t}\right\}$ :

$$
\mathcal{L} g(y)=\sum_{1 \leq k, l \leq d} q^{k l}(y) g_{y_{k} y_{l}}(y)+\sum_{1 \leq k \leq d} B^{k}(y) g_{y_{k}}(y)
$$

with $q=\frac{1}{2} \sigma \sigma^{*}$,

- and

$$
\mathcal{A}^{\varepsilon} h(x)=\operatorname{div}\left(a\left(\frac{x}{\varepsilon}, y\right) \nabla h(x)\right) .
$$

$\mathcal{A}$ will denote $\mathcal{A}^{\varepsilon}$ for $\varepsilon=1$.

Note that, applied to a function $f(z, y), \mathcal{L}$ acts on the function $y \mapsto f(z, y)$ for $z$ fixed, and $\mathcal{A}^{\varepsilon}$ acts on the function $z \mapsto f(z, y)$ for $y$ fixed.

\subsection{Notations}

- In $\mathbb{R}^{n}, x \cdot x^{\prime}$ will denote the scalar product and $|\cdot|$ the corresponding norm.

$\mathrm{RR} \mathrm{n}^{\circ} 3520$ 
- In the space $L^{2}\left(\mathbb{R}^{n}\right),(\cdot, \cdot)$ will denote the inner product, and $\|\cdot\|$ the norm.

- For a function $(z, y) \mapsto f(z, y)$, we use the following notations:

$$
\begin{aligned}
\overline{\langle f(\cdot, \cdot)\rangle} & =\int_{\mathbb{T}_{n}} \int_{\mathbb{R}^{d}} f(z, y) \rho(y) d y d z \\
\langle f(\cdot, y)\rangle & =\int_{\mathbb{T}_{n}} f(z, y) d z \\
\overline{f(z, \cdot)} & =\int_{\mathbb{R}^{d}} f(z, y) \rho(y) d y
\end{aligned}
$$

here and in what follows $\rho(y)$ stands for the density of invariant measure of process $\xi_{s}$. The question of the existence of invariant measure and the properties of $\rho(y)$ will be discussed later.

- For a function or process $(t, x) \mapsto u(t, x), u(t)$ will denote an application

$x \mapsto u(t, x)$. Hence $\|u(t)\|$ is $\left(\int_{\mathbb{R}^{n}}|u(t, x)|^{2} d x\right)^{1 / 2}$. This notation is also used for $u^{\varepsilon}(t, x)$ and for the gradient $\nabla u^{\varepsilon}(t, x)$. We use, as well, the contracted notation:

$$
c^{\varepsilon}=c\left(\frac{x}{\varepsilon}, \xi_{t / \varepsilon^{\alpha}}\right), \quad a^{\varepsilon}=a\left(\frac{x}{\varepsilon}, \xi_{t / \varepsilon^{\alpha}}\right), \quad a^{\varepsilon, i j}=a^{i j}\left(\frac{x}{\varepsilon}, \xi_{t / \varepsilon^{\alpha}}\right)
$$

and for a generic function $g(z, y): g^{\varepsilon}=g\left(\frac{x}{\varepsilon}, \xi_{t / \varepsilon^{\alpha}}\right)$.

\subsection{Hypotheses}

In this section, we provide the precise assumptions on the coefficient of (1) and on the process $\xi_{t}$.

Hypothesis 2.1 The coefficients $a, c$, and $q$ are uniformly bounded as well as their derivatives: there exists $C_{1}>0$ such that for all $(z, y) \in \mathbb{T}^{n} \times \mathbb{R}^{d}$

$$
\begin{aligned}
\left|a^{i j}(z, y)\right|+\left|\nabla_{z} a^{i j}(z, y)\right|+\left|\nabla_{y} a^{i j}(z, y)\right| & \leq C_{1} \\
|c(z, y)|+\left|\nabla_{z} c(z, y)\right|+\left|\nabla_{y} c(z, y)\right| & \leq C_{1} \\
\left|q^{k l}(y)\right|+\left|\nabla_{y} q^{i j}(y)\right| & \leq C_{1}
\end{aligned}
$$


for all $1 \leq i, j \leq n, 1 \leq k, l \leq d\left({ }^{1}\right)$. The vector function $B$ as well as its derivatives satisfy polynomial growth condition:

$$
|B(y)|+\left|\nabla_{y} B(y)\right| \leq C_{1}(1+|y|)^{\mu_{1}}
$$

for some $\mu_{1} \geq 0$.

Hypothesis 2.2 Operators $\mathcal{L}$ and $\mathcal{A}$ are uniformly elliptic: there exists a constant $C_{2}>0$ such that for all $(z, y) \in \mathbb{T}^{n} \times \mathbb{R}^{d}$

$$
\begin{aligned}
& C_{2}\left|z^{\prime}\right|^{2} \leq\left(a(z, y) z^{\prime}\right) \cdot z^{\prime}, \forall z^{\prime} \in \mathbb{R}^{n}, \\
& C_{2}\left|y^{\prime}\right|^{2} \leq\left(q(z, y) y^{\prime}\right) \cdot y^{\prime}, \forall y^{\prime} \in \mathbb{R}^{d} .
\end{aligned}
$$

Hypothesis 2.3 There exist constants $\mu>-1, R_{1}>0$ and $C_{3}>0$ such that

$$
B(y) \cdot \frac{y}{|y|} \leq-C_{3}|y|^{\mu}, \quad \forall y \text { s.t. }|y|>R_{1} \text {. }
$$

Under Hypotheses 2.1, 2.2, and 2.3, process $\left\{\xi_{t}\right\}$ admits the unique invariant measure with smooth density $\rho(y)$ given by:

$$
\mathcal{L}^{*} \rho=0 \text { on } \mathbb{R}^{d} \text {, and } \int_{\mathbb{R}^{d}} \rho(y) d y=1 .
$$

Moreover, the density $\rho(\cdot)$ decays faster than any negative power of $|y|$ as $|y| \rightarrow \infty$ (see [13]). In fact, the following bound holds

$$
\rho(y) \leq c_{1} \exp \left(-c|y|^{1+\mu}\right), c>0 .
$$

Hypothesis 2.4 We will suppose that

$$
\overline{\langle c(\cdot, \cdot)\rangle}=0 .
$$

This hypothesis is, in fact, not a restriction. Indeed, considering the new unknown function $\tilde{u}^{\varepsilon}(t, x)=e^{-t \overline{\langle c\rangle} / \varepsilon} u^{\varepsilon}(t, x)$, one can always achieve the relation $\overline{\langle c\rangle}=0$.

\footnotetext{
${ }^{1}$ Notations : $\nabla_{z}, \nabla_{y}$ are the space gradient with respect to $z$ and $y$ respectively. When there is no ambiguity about the argument of the function we use the notation $\nabla$, for example $\nabla u^{\varepsilon}(t, x)$ is $\nabla_{x} u^{\varepsilon}(t, x)$.
}

$\mathrm{RR} \mathrm{n}^{\circ} 3520$ 


\subsection{Auxiliary results}

Let $\left\{\left(\zeta_{t}, \xi_{t}\right)\right\}$ be the diffusion process associated to the infinitesimal generator $\mathcal{A}+\mathcal{L}$, and $L_{\rho}^{2}\left(\mathbb{T}^{n} \times \mathbb{R}^{d}\right)$ denote the weighted space with the norm:

$$
\|f\|_{\rho}^{2}=\int_{\mathbb{T}^{n}} \int_{\mathbb{R}^{d}} f(z, y)^{2} \rho(y) d y d z .
$$

Also, we introduce the spaces:

$$
\begin{aligned}
\bar{L}_{\rho}^{2}\left(\mathbb{T}^{n} \times \mathbb{R}^{d}\right) & =\left\{f \in L_{\rho}^{2}\left(\mathbb{T}^{n} \times \mathbb{R}^{d}\right) ; \overline{\langle f(\cdot, \cdot)\rangle}=0\right\} \\
\bar{H}_{\rho}^{1}\left(\mathbb{T}^{n} \times \mathbb{R}^{d}\right) & =\left\{f \in \bar{L}_{\rho}^{2}\left(\mathbb{T}^{n} \times \mathbb{R}^{d}\right) ;\left|\nabla_{x} f\right|+\left|\nabla_{z} f\right| \in L_{\rho}^{2}\left(\mathbb{T}^{n} \times \mathbb{R}^{d}\right)\right\} .
\end{aligned}
$$

The next statement is a version of Theorem 1 from [13] and can be proven exactly in the same way.

Lemma 2.5 Let $f \in \bar{L}_{\rho}^{2}\left(\mathbb{T}^{n} \times \mathbb{R}^{d}\right)$, and assume that

$$
|f(z, y)| \leq C_{5}\left(1+|y|^{p}\right) \forall(z, y) \in \mathbb{T}^{n} \times \mathbb{R}^{d}
$$

for some constants $C_{5}>0$ and $p \in \mathbb{N}$. Then the equation:

$$
(\mathcal{A}+\mathcal{L}) u(z, y)=f(z, y)
$$

does have a unique solution $u \in \bar{H}_{\rho}^{1}\left(\mathbb{T}^{n} \times \mathbb{R}^{d}\right)$ and the estimate

$$
|u(z, y)| \leq C_{6}\left(1+|y|^{p_{1}}\right) \forall(z, y) \in \mathbb{T}^{n} \times \mathbb{R}^{d}
$$

holds; moreover, $p_{1}$ depends only on $p$ and $\mu$ and the constant $C_{6}$ depends only on $C_{5}$ and $p$ and $\mu$ (we assume that the dimensions are fixed).

If, in addition, there exists $N>0$ such that for all $n_{1}, n_{2} \in \mathbb{N}$ with $n_{1}+n_{2} \leq$ $N$ we have:

$$
\left|\partial_{z}^{n_{1}} \partial_{y}^{n_{2}} f(z, y)\right| \leq C_{5}\left(1+|y|^{p}\right) \forall(z, y) \in \mathbb{T}^{n} \times \mathbb{R}^{d}
$$

then

$$
\left|\partial_{z}^{n_{1}} \partial_{y}^{n_{2}} u(z, y)\right| \leq C_{6}\left(1+|y|^{p_{1}}\right) \forall(z, y) \in \mathbb{T}^{n} \times \mathbb{R}^{d}
$$


Applying the technique developed in [13], Proposition 2, leads to the following statement:

Proposition 2.6 For any fixed $T>0, p>0, \alpha>0$, and $\beta>0$ :

$$
\lim _{\varepsilon \downarrow 0} E\left(\sup _{t \leq T} \varepsilon^{\beta}\left|\xi_{\frac{t}{\varepsilon^{\alpha}}}\right|^{p}\right)=0 .
$$

$\mathrm{RR} \mathrm{n}^{\circ} 3520$ 


\section{Main results}

Here we formulate the main results of the paper; the proof will be given in the following section.

It should be noted that for $\alpha \leq 2$, we obtain the weak convergence of the distribution of $u^{\varepsilon}(t, x)$ to the nontrivial limit distribution being the solution of a proper martingale problem while for $\alpha>2$, the limit distribution is a $\delta$-type distribution concentrated on the solution of the Cauchy problem for the limit parabolic equation with constant coefficients.

Let $L_{w}^{2}\left(\mathbb{R}^{n}\right)$ denote the space $L^{2}\left(\mathbb{R}^{n}\right)$ endowed with the weak topology. Define:

$$
\Omega_{T}=L_{w}^{2}\left((0, T) ; H^{1}\left(\mathbb{R}^{n}\right)\right) \cap \mathcal{C}\left([0, T] ; L_{w}^{2}\left(\mathbb{R}^{n}\right)\right)
$$

endowed with supremum of the topology of uniform convergence over the space $\mathcal{C}\left([0, T] ; L_{w}^{2}\left(\mathbb{R}^{n}\right)\right)$ and weak topology over the space $L^{2}\left((0, T) ; H^{1}\left(\mathbb{R}^{n}\right)\right) . \Omega_{T}$ is a Lusin and regular space, denote by $\mathcal{F}$ its Borel $\sigma$-field.

For any $\varepsilon>0$, let $Q^{\varepsilon}$ be the Radon probability measure on $\left(\Omega_{T}, \mathcal{F}\right)$ which is defined by the law of $\left\{u^{\varepsilon}(t) ; 0 \leq t \leq T\right\}$. The asymptotic behavior of $u^{\varepsilon}$, as $\varepsilon \downarrow 0$, depends on whether $\alpha<2, \alpha=2$, or $\alpha>2$.

\subsection{Case $\alpha<2$}

Let $\hat{Q}$ denote the probability measure on $\left(\Omega_{T}, \mathcal{F}\right)$ associated with the solution of the following (limit) SPDE:

$$
d \hat{u}(t, x)=[\operatorname{div}(\hat{a} \nabla \hat{u}(t, x))+\hat{c} \hat{u}(t, x)] d t+\lambda \hat{u}(t, x) d \hat{W}_{t}
$$

with initial condition $\hat{u}(0, x)=u_{0}(x)$, where $(t, x) \in[0, T] \times \mathbb{R}^{n},\left\{\hat{W}_{t}\right\}$ is standard Brownian motion in $\mathbb{R}$ and:

$$
\begin{aligned}
\hat{a} & =\overline{\left\langle a\left(I+\nabla_{z} \Psi\right)\right\rangle}, \\
\hat{c} & =\overline{\langle G c\rangle} \\
\lambda^{2} & =2 \overline{q \nabla G \cdot \nabla G},
\end{aligned}
$$


and the functions $G \in \bar{H}_{\rho}^{1}\left(\mathbb{R}^{d}\right)$ and $\Psi^{i} \in \bar{H}_{\rho}^{1}\left(\mathbb{T}^{n} \times \mathbb{R}^{d}\right)$ are the solutions of the equations:

$$
\begin{aligned}
\mathcal{L} G(y) & =-\langle c(\cdot, y)\rangle, \\
\mathcal{A} \Psi^{i}(z, y) & =-\sum_{j=1}^{n} a_{z_{j}}^{i j}(z, y),
\end{aligned}
$$

for $(z, y) \in \mathbb{T}^{n} \times \mathbb{R}^{d}$ and $1 \leq i \leq n$.

Theorem 3.1 Let $\alpha<2$, then under Hypotheses 2.1, 2.2, 2.3, and 2.4, the law $Q^{\varepsilon}$ of the solution $u^{\varepsilon}$ of Equations (1)-(2) converges, as $\varepsilon \downarrow 0$, in space $\Omega_{T}$ to the law $\hat{Q}$ of the solution $\hat{u}$ of Equation (8).

\subsection{Case $\alpha=2$}

Let $\hat{Q}$ denote the probability measure associated with the solution of the following (limit) SPDE:

$$
d \hat{u}(t, x)=[\operatorname{div}(\hat{a} \nabla \hat{u}(t, x))-\hat{b} \cdot \nabla \hat{u}(t, x)+\hat{c} \hat{u}(t, x)] d t+\lambda \hat{u}(t, x) d \hat{W}_{t},
$$

with initial condition $\hat{u}(0, x)=u_{0}(x)$, where $(t, x) \in[0, T] \times \mathbb{R}^{n},\left\{\hat{W}_{t}\right\}$ is a standard Brownian motion in $\mathbb{R}$, and:

$$
\begin{aligned}
\hat{a} & =\overline{\left\langle a\left(I+\nabla_{z} \Psi\right)\right\rangle}, \\
\hat{b} & =\overline{\left\langle\Psi c+a \nabla_{z} G\right\rangle}, \\
\hat{c} & =\overline{\langle G c\rangle}, \\
\lambda^{2} & =2 \overline{q\left\langle\nabla_{y} G\right\rangle \cdot\left\langle\nabla_{y} G\right\rangle},
\end{aligned}
$$

and the functions $G, \Psi^{j} \in \bar{H}_{\rho}^{1}\left(\mathbb{T}^{n} \times \mathbb{R}^{d}\right)$ are the solutions of the equations:

$$
\begin{aligned}
(\mathcal{A}+\mathcal{L}) G(z, y) & =-c(z, y) \\
(\mathcal{A}+\mathcal{L}) \Psi^{j}(z, y) & =-\sum_{i=1}^{n} a_{z_{i}}^{i j}(z, y)
\end{aligned}
$$

for $(z, y) \in \mathbb{T}^{n} \times \mathbb{R}^{d}$ and $1 \leq j \leq n$.

$\mathrm{RR} \mathrm{n}^{\circ} 3520$ 
Theorem 3.2 For $\alpha=2$, under Hypotheses 2.1, 2.2, 2.3, and 2.4, the law $Q^{\varepsilon}$ of the solution $u^{\varepsilon}$ of Equations (1)-(2) converges, as $\varepsilon \downarrow 0$, in space $\Omega_{T}$ to the law $\hat{Q}$ of the solution $\hat{u}$ of Equation(11).

\subsection{Case $\alpha>2$}

Let $\hat{u}$ be the solution of the following Cauchy problem:

$$
\hat{u}_{t}(t, x)=\operatorname{div}(\hat{a} \nabla \hat{u}(t, x))+\hat{c} \hat{u}(t, x), \quad \hat{u}(0, x)=u_{0}(x)
$$

with $(t, x) \in[0, T] \times \mathbb{R}^{n}$ and:

$$
\hat{a}=\left\langle\bar{a}\left(I+\nabla_{z} \Psi\right)\right\rangle, \quad \hat{c}=\langle G \bar{c}\rangle,
$$

where the functions $G, \Psi^{i} \in \bar{H}^{1}\left(\mathbb{T}^{n}\right)$ are solutions of equations:

$$
\begin{aligned}
\overline{\mathcal{A}} G(z) & =-\overline{c(z, \cdot)} \\
\overline{\mathcal{A}} \Psi^{i}(z) & =-\sum_{j=1}^{n} \overline{a_{z_{j}}^{i j}(z, \cdot)}
\end{aligned}
$$

for $z \in \mathbb{T}^{n}$ and $1 \leq i \leq n$, where the operator $\overline{\mathcal{A}}$ is defined by:

$$
\overline{\mathcal{A}} f(z)=\operatorname{div}(\overline{a(z, \cdot)} \nabla f(z)) .
$$

Theorem 3.3 For $\alpha>2$, under Hypotheses 2.1, 2.2, 2.3, and 2.4, the solution $u^{\varepsilon}$ of Equations (1)-(2) converges in probability in the space $\Omega_{T}$ to the solution $\hat{u}$ of the limit problem (14).

Corollary 3.4 For $\alpha>2$, under Hypotheses 2.1, 2.2, 2.3, and 2.4, we have

$$
P-\lim _{\varepsilon \downarrow 0}\left\|u^{\varepsilon}-\hat{u}\right\|_{L^{2}\left((0, T) \times \mathbb{R}^{n}\right)}=0
$$

where $u^{\varepsilon}$ (resp. $\left.\hat{u}\right)$ is the solution of Equations (1)-(2) (resp. (14)). 


\subsection{Comments}

Comparison with systems without potential or without "noise input" It is interesting to compare the limit problems (8), (11), (14) above with the limit problems for the equation without potential:

$$
u_{t}(t, x)=\operatorname{div}\left(a\left(\frac{x}{\varepsilon}, \xi_{t / \varepsilon^{\alpha}}\right) \nabla u(t, x)\right)
$$

and the equation "without noise input":

$$
u_{t}(t, x)=\operatorname{div}\left(a\left(\frac{x}{\varepsilon}\right) \nabla u(t, x)\right)+\frac{1}{\varepsilon} c\left(\frac{x}{\varepsilon}\right) u(t, x) .
$$

According to [8], the absence of the potential in (19) always leads to the deterministic form of homogenized problem:

$$
\bar{u}_{t}(t, x)=\sum_{1 \leq i, j \leq n} \bar{a}^{i j} \frac{\partial^{2}}{\partial x_{i} \partial x_{j}} \bar{u}(t, x),
$$

this operator involves neither stochastic nor lower order terms. The limit problem for (20) takes the following form:

$$
\bar{u}_{t}(t, x)=\sum_{1 \leq i, j \leq n} \bar{a}^{i j} \frac{\partial^{2}}{\partial x_{i} \partial x_{j}} \bar{u}(t, x)+\bar{c} \bar{u}(t, x)
$$

where $\bar{c}$ is the so-called "strange term".

Comment on the limiting equation (11) The appearance of the first order term $\hat{b} \cdot \nabla$ in the drift part of the limit problem (11) is of special interest. It should be shown that this first order term is not necessarily null.

To this end, let propose an example with $n=1$ and $d=2$. We take $q^{k l}=\delta_{k l}$ in (4) and choose:

$$
B(y)=-2\left(\begin{array}{l}
y_{1}+\nu y_{2} \\
y_{2}-\nu y_{1}
\end{array}\right)
$$

where $\nu>0$. One can easily check that the density $\rho(y)$ of the invariant measure of $\xi_{t}$ is given, under this choice of $q^{k l}$ and $B(y)$, by the formula:

$$
\rho(y)=c e^{-|y|^{2}}
$$

$\mathrm{RR} \mathrm{n}^{\circ} 3520$ 
here $c$ is a normalization constant.

We consider the one--dimensional case (w.r.t. $z$ ):

$$
\hat{b}=\int_{\mathbb{T}^{1}} \int_{\mathbb{R}^{2}}\left[\Psi(z, y) c(z, y)+a(z, y) G_{z}(z, y)\right] \rho(y) d z d y .
$$

Integrating by parts and taking into account Equations (12)-(13), we obtain:

$$
\hat{b}=\int_{\mathbb{T}^{1}} \int_{\mathbb{R}^{2}}(\Psi \mathcal{L} G-G \mathcal{L} \Psi)(z, y) \rho(y) d z d y .
$$

here we also used the divergent form and the symmetry of the operator $\mathcal{A}$.

Finally, in view of (6), after integrating by parts, we get:

$$
\begin{aligned}
\hat{b} & =-\int_{\mathbb{T}^{1}} \int_{\mathbb{R}^{2}}\left[\left(\nabla_{y} \Psi\right) \cdot G\left(\nabla_{y} \rho-B \rho\right)\right](z, y) d z d y \\
& =-\nu \int_{\mathbb{T}^{1}} \int_{\mathbb{R}^{2}}\left(\nabla_{y} \Psi\right)(z, y) \cdot G(z, y)\left(\begin{array}{c}
y_{2} \\
y_{1}
\end{array}\right) d z d y .
\end{aligned}
$$

Denote $k(y)=(\mathcal{L}-1) \sin \left(y_{1}\right)$ and construct the operator $\mathcal{A}$ to be a small perturbation of operator with constant coefficient:

$$
a(z, y)=1+\aleph d(z, y)
$$

where $\aleph$ is a small parameter and $d(z, y)=\sin (z) k(y)$.

It is easy to see that the function $\Psi^{1}(z, y)=\cos (z) \sin \left(y_{1}\right)$ solves the following auxiliary problem:

$$
\left(\frac{d^{2}}{d z^{2}}+\mathcal{L}\right) \Psi^{1}(z, y)=-\cos (z) k(y) .
$$

Now, substituting the function $\aleph \Psi^{1}(z, y)$ in the equation (13) which in our particular case reads

$$
\left(\frac{d}{d z} a(z, y) \frac{d}{d z}+\mathcal{L}\right) \Psi(z, y)=-\aleph \frac{d}{d z} d(z, y)=-\aleph \cos (z) k(y)
$$

and taking into account the coerciveness of the latter problem, we get

$$
\left\|\Psi-\aleph \Psi^{1}\right\|=O\left(\aleph^{2}\right) \text { as } \aleph \downarrow 0 .
$$


Thus, it suffices to show that the integral:

$$
\begin{aligned}
\int_{\mathbb{T}^{1}} \int_{\mathbb{R}^{2}}\left(\nabla_{y} \Psi^{1}\right)(z, y) & \cdot G(z, y)\left(\begin{array}{c}
y_{2} \\
y_{1}
\end{array}\right) e^{-|y|^{2}} d z d y= \\
= & \int_{\mathbb{T}^{1}} \int_{\mathbb{R}^{2}} \cos (z) y_{2} \cos \left(y_{1}\right) G(z, y) e^{-|y|^{2}} d z d y
\end{aligned}
$$

is not equal to zero under the proper choice of $G(z, y)$.

To do so one can construct a smooth nonnegative function $G(z, y)$ with a small compact support concentrated near the point $\left(z^{0}, y^{0}\right)$ such that:

$$
y_{2}^{0} \cos \left(z^{0}\right) \cos \left(y_{1}^{0}\right) e^{-\left|y^{0}\right|^{2}} \neq 0
$$

say $\left(z^{0}, y^{0}\right)=\left(\frac{\pi}{4},\left(\frac{\pi}{4}, \frac{\pi}{4}\right)\right)$.

$\mathrm{RR} \mathrm{n}^{\circ} 3520$ 


\section{Proof of the main results}

\subsection{Decomposition of the solution $u^{\varepsilon}-$ Auxiliary ho- mogenization problem}

The expectation of the $L^{2}$ or $L^{2}\left((0, T) ; H^{1}\left(\mathbb{R}^{n}\right)\right)$-norms of $u^{\varepsilon}$ in general does not admit uniform in $\varepsilon$ estimates. Thus, in order to obtain the compactness of $u^{\varepsilon}$ we need an approach that does not rely on estimates of the expectation.

In this section we decompose $u^{\varepsilon}$ into the product of the exponent of given function and of new unknown function $v^{\varepsilon}(x, t)$ :

$$
u^{\varepsilon}(t, x)=\exp \left(\frac{1}{\varepsilon^{1 \wedge \frac{\alpha}{2}}} \int_{0}^{t}\left\langle c\left(\cdot, \xi_{\frac{s}{\varepsilon^{\alpha}}}\right)\right\rangle d s\right) v^{\varepsilon}(t, x) .
$$

Direct calculations show that the function $v^{\varepsilon}$ satisfies the following equation

$$
\begin{aligned}
v_{t}^{\varepsilon}(t, x) & =\operatorname{div}\left[a\left(\frac{x}{\varepsilon}, \xi_{\frac{t}{\varepsilon \alpha}}\right) \nabla v^{\varepsilon}(t, x)\right]+\frac{1}{\varepsilon^{1 \wedge \frac{\alpha}{2}}} \tilde{c}\left(\frac{x}{\varepsilon}, \xi_{\frac{t}{\varepsilon \alpha}}\right) v^{\varepsilon}(t, x), \\
v^{\varepsilon}(0, x) & =u_{0}(x), x \in \mathbb{R}^{n}, t \in[0, T]
\end{aligned}
$$

where $\tilde{c}(z, y)=c(z, y)-\langle c(\cdot, y)\rangle$.

In the following sections we show that $v^{\varepsilon}$ converges in probability in the space $\Omega_{T}$ to nonrandom function being the solution of Cauchy problem with constant coefficient.

\subsection{Tightness result for auxiliary homogenization prob- lem}

This section deals with the tightness of the family of distributions associated with solutions $v^{\varepsilon}$ of problem (25)-(26). We start by obtaining uniform estimates for $v^{\varepsilon}$.

Proposition 4.1 The inequalities

$$
\begin{aligned}
& \sup _{t \leq T}\left\|v^{\varepsilon}(t)\right\|^{2} \leq c, \\
& \int_{0}^{T}\left\|\nabla v^{\varepsilon}(s)\right\|^{2} d s \leq c,
\end{aligned}
$$


hold uniformly in $\varepsilon>0$. For any test function $\varphi(x)$ the inequality

$$
\left|\left(\varphi, v^{\varepsilon}(t)-v^{\varepsilon}(s)\right)\right| \leq c|t-s|^{1 / 2}
$$

holds uniformly in $\varepsilon>0$.

Proof Multiplying Equation (25) by $v^{\varepsilon}$ and integrating by parts we get:

$$
\begin{aligned}
\frac{1}{2}\left\|v^{\varepsilon}\right\|_{t}^{2} & =-\left(a^{\varepsilon} \nabla v^{\varepsilon}, \nabla v^{\varepsilon}\right)+\varepsilon^{-(1 \wedge \alpha / 2)}\left(\tilde{c}^{\varepsilon} v^{\varepsilon}, v^{\varepsilon}\right) \\
& =-\left(a^{\varepsilon} \nabla v^{\varepsilon}, \nabla v^{\varepsilon}\right)+2 \varepsilon^{\gamma}\left(\kappa^{\varepsilon} v^{\varepsilon}, \nabla v^{\varepsilon}\right),
\end{aligned}
$$

where $\gamma=1-(1 \wedge \alpha / 2)$ and $\kappa(z, y)$ is $z$-periodic vector-function defined by the relation

$$
\operatorname{div}_{z} \kappa(z, y)=\tilde{c}(z, y) .
$$

With the help of the Cauchy-Buniakovski inequality after simple transformation we obtain:

$$
\begin{aligned}
\frac{1}{2}\left\|v^{\varepsilon}\right\|^{2} & \leq \frac{1}{2}\left\|u_{0}\right\|^{2}-\int_{0}^{t}\left(a^{\varepsilon} \nabla v^{\varepsilon}, \nabla v^{\varepsilon}\right) d s+\lambda \int_{0}^{t}\left\|\nabla v^{\varepsilon}\right\|^{2} d s+\frac{c}{\lambda} \int_{0}^{t}\left\|v^{\varepsilon}\right\|^{2} d s \\
& \leq \frac{1}{2}\left\|u_{0}\right\|^{2}-\Lambda \int_{0}^{t}\left\|\nabla v^{\varepsilon}\right\|^{2} d s+c_{1} \int_{0}^{t}\left\|v^{\varepsilon}\right\|^{2} d s
\end{aligned}
$$

for some $\Lambda>0$. Now (E1)-(E2) follows from Gronwall's lemma.

For any test function $\varphi$ we have

$$
\begin{aligned}
\left|\left(\varphi, v^{\varepsilon}(t)-v^{\varepsilon}(s)\right)\right| & \leq \int_{s}^{t}\left(\nabla \varphi, a^{\varepsilon} \nabla v^{\varepsilon}\right) d r+\varepsilon^{\gamma} \int_{s}^{t} \mid\left(\kappa^{\varepsilon}, \nabla\left(\varphi v^{\varepsilon}\right) \mid d r\right. \\
& \leq c|t-s|^{1 / 2}
\end{aligned}
$$

This completes the proof.

For any $\varepsilon>0$ denote $\tilde{Q}^{\varepsilon}$ a Radon probability measure on $\left(\Omega_{T}, \mathcal{F}\right)$ defined as the law of $\left\{v^{\varepsilon}(t) ; 0 \leq t \leq T\right\}$ in $\Omega_{T}$. Proposition 4.1 implies the tightness of the family $\left\{\tilde{Q}^{\varepsilon}\right\}$ in $\Omega_{T}$.

$\mathrm{RR} \mathrm{n}^{\circ} 3520$ 


\subsection{Description of homogenized operator for auxiliary problem - Convergence in probability}

In this section we prove the convergence of $v^{\varepsilon}$ in probability and show that the limit function satisfies parabolic equation with constant coefficients. We study the cases $\alpha>2, \alpha=2$ and $\alpha<2$ separately.

Case $\alpha<2$

Let $\hat{v}(t, x)$ be the solution of the following Cauchy problem

$$
\hat{v}_{t}=\operatorname{div}(\hat{a} \nabla \hat{v}), \hat{v}(x, 0)=u_{0}(x),
$$

$\hat{a}$ has been defined in Section 3.1.

Proposition 4.2 For any test function $\varphi(x, t) \in C^{\infty}\left([0, T] ; C_{0}^{\infty}\left(R^{n}\right)\right)$ the following limit relation holds

$$
\begin{aligned}
\lim _{\varepsilon \downarrow 0} E \sup _{t \leq T} \mid & \left(\varphi(t), v^{\varepsilon}(t)\right)-\left(\varphi(0), u_{0}\right)-\int_{0}^{t}\left(\varphi_{s}(s), v^{\varepsilon}(s)\right) d s \\
& -\int_{0}^{t}\left(\sum_{i j} \hat{a}^{i j} \varphi_{x_{i} x_{j}}(s), v^{\varepsilon}(s)\right) d s \mid=0
\end{aligned}
$$

Proof Let us introduce a process

$$
H^{\varepsilon}(t)=\left(\varphi, v^{\varepsilon}(t)\right)+\sum_{l=0}^{K} \varepsilon^{1+l \delta} \sum_{k=1}^{n}\left(\Psi^{k l}\left(\dot{\bar{\varepsilon}}, \xi_{t / \varepsilon^{\alpha}}\right) \varphi_{x_{k}}, v^{\varepsilon}(t)\right)
$$

where $\delta=2-\alpha, K=[1 / \delta]+1$, and $z$-periodic functions $\Psi^{k l}(z, y) \in \bar{H}_{\rho}^{1}\left(\mathbb{T}^{n} \times\right.$ $\mathbb{R}^{n}$ ) are defined as the solutions of the following equations:

$$
\left\{\begin{array}{lll}
\mathcal{A} \Psi^{k 0}(z, y)=-\sum_{j} a_{z_{j}}^{k j}(z, y), & k=1, \ldots, n \\
\mathcal{A} \Psi^{k l}(z, y)=-\mathcal{L} \Psi^{k(l-1)}(z, y), & l=1, \ldots, K
\end{array}\right.
$$

From Proposition 4.1, (E1)-(E2), and Proposition 2.6 we have

$$
\lim _{\varepsilon \rightarrow 0} E \sup _{t \leq T}\left|\left(\varphi, v^{\varepsilon}\right)-H^{\varepsilon}(t)\right|=0 .
$$


Now, in order to prove (29), we apply Ito's formula to the process $H^{\varepsilon}(t)$ :

$$
\begin{gathered}
d H^{\varepsilon}=\sum_{i j}\left(a^{\varepsilon, i j} \varphi_{x_{i} x_{j}}, v^{\varepsilon}\right) d t+\varepsilon^{-1} \sum_{i j}\left(a_{z_{j}}^{\varepsilon, i j} \varphi_{x_{i}}, v^{\varepsilon}\right) d t \\
+\varepsilon^{-\alpha / 2}\left(\tilde{c}^{\varepsilon} \varphi, v^{\varepsilon}\right) d t \\
+\sum_{l} \varepsilon^{1+l \delta} \sum_{k} \\
\quad\left[\varepsilon^{-\alpha}\left(\mathcal{L} \Psi^{k l} \varphi_{x_{k}}, v^{\varepsilon}\right) d t+\varepsilon^{-\alpha / 2}\left(\nabla_{y} \Psi^{k l} \varphi_{x_{k}}, v^{\varepsilon}\right) \cdot \sigma^{\varepsilon} d W_{t}\right. \\
\quad+\varepsilon^{-2}\left(\mathcal{A} \Psi^{k l} \varphi_{x_{k}}, v^{\varepsilon}\right) d t+\varepsilon^{-\alpha / 2}\left(\Psi^{k l} \tilde{c}^{\varepsilon} \varphi_{x_{k}}, v^{\varepsilon}\right) d t \\
\quad+\varepsilon^{-1} \sum_{i j}\left(\left(a^{\varepsilon, i j} \Psi^{k l}\right)_{z_{i}} \varphi_{x_{k} x_{j}}+a^{\varepsilon, i j} \Psi_{z_{j}}^{k l} \varphi_{x_{k} x_{i}}, v^{\varepsilon}\right) d t \\
\left.+\sum_{i j}\left(a^{\varepsilon, i j} \Psi^{k l} \varphi_{x_{k} x_{i} x_{j}}, v^{\varepsilon}\right) d t\right] .
\end{gathered}
$$

Taking into account (31) after simple transformation we get

$$
\begin{aligned}
d H^{\varepsilon}=\sum_{i j}\left(a^{\varepsilon, i j} \varphi_{x_{i} x_{j}}, v^{\varepsilon}\right) d t+\varepsilon^{\delta / 2}\left(\kappa^{\varepsilon}, \nabla\left(\varphi v^{\varepsilon}\right)\right) d t \\
\quad+\sum_{k} \sum_{i j}\left(\left(a^{\varepsilon, i j} \Psi^{k 0}\right)_{z_{i}} \varphi_{x_{k} x_{j}}+a^{\varepsilon, i j} \Psi_{z_{j}}^{k 0} \varphi_{x_{k} x_{i}}, v^{\varepsilon}\right) d t \\
\quad+\left(\varphi_{t}, v^{\varepsilon}\right) d t+\varepsilon^{\delta / 2} d R^{\varepsilon}(t)
\end{aligned}
$$

where $E \sup _{t \leq T}\left|R^{\varepsilon}(t)\right| \leq c$. Therefore,

$$
\begin{aligned}
\left(\varphi, v^{\varepsilon}\right)-\left(\varphi, u_{0}\right)- & \int_{0}^{t}\left(\varphi_{s}, v^{\varepsilon}\right) d s-\int_{0}^{t} \sum_{i j}\left(\hat{a}^{i j} \varphi_{x_{i} x_{j}}, v^{\varepsilon}\right) d s \\
=\int_{0}^{t}\left[\sum _ { i j } \left(a^{\varepsilon, i j}\right.\right. & \left.\varphi_{x_{i} x_{j}}, v^{\varepsilon}\right) \\
& +\sum_{k} \sum_{i j}\left(\left(a^{\varepsilon, i j} \Psi^{k 0}\right)_{z_{i}} \varphi_{x_{k} x_{j}}+a^{\varepsilon, i j} \Psi_{z_{j}}^{k 0} \varphi_{x_{k} x_{i}}, v^{\varepsilon}\right) \\
& \left.-\sum_{i j}\left(\hat{a}^{i j} \varphi_{x_{i} x_{j}}, v^{\varepsilon}\right)\right] d s+\varepsilon^{\delta / 2} R^{\varepsilon}(t) ;
\end{aligned}
$$

where we have also added and subtracted the term $\sum_{i j}\left(\hat{a}^{i j} \varphi_{x_{i} x_{j}}, v^{\varepsilon}\right) d t$. The integral in the right hand side of the last relation can be represented in the form

$$
\int_{0}^{t} \sum_{m}\left(\left[h^{m}\left(\left(\dot{\bar{\varepsilon}}, \xi_{s / \varepsilon^{\alpha}}\right)-\overline{\left\langle h^{m}\right\rangle}\right] \tilde{\varphi}^{m}, v^{\varepsilon}\right) d s\right.
$$

where $\tilde{\varphi}^{m}$ denotes one of the functions $\varphi, \varphi_{x_{i}}, \varphi_{x_{i} x_{j}}$ and $h^{m}\left(\frac{x}{\varepsilon}, \xi_{t / \varepsilon^{\alpha}}\right)$ denotes one of the functions $a^{i j}\left(\frac{x}{\varepsilon}, \xi_{t / \varepsilon^{\alpha}}\right), \Psi^{k 0}\left(\frac{x}{\varepsilon}, \xi_{t / \varepsilon^{\alpha}}\right), \Psi_{z_{i}}^{k 0}\left(\frac{x}{\varepsilon}, \xi_{t / \varepsilon^{\alpha}}\right)$. To complete the proof of Proposition 4.2 it remains to show that

$$
P-\lim _{\varepsilon \downarrow 0} \sup _{t \leq T}\left|\int_{0}^{t}\left(\left[h^{m}\left(\dot{\bar{\varepsilon}}, \xi_{s / \varepsilon^{\alpha}}\right)-\overline{\left\langle h^{m}\right\rangle}\right] \tilde{\varphi}^{m}, v^{\varepsilon}(s)\right) d s\right|=0 .
$$

$\mathrm{RR} \mathrm{n}^{\circ} 3520$ 
Proposition 4.3 For any $z$-periodic function $(z, y) \mapsto h(z, y)$ such that $\overline{\langle h\rangle}=$ 0 and $|h| \leq c(1+|y|)^{\theta}$ for some $\theta$, we have:

$$
P-\lim _{\varepsilon \downarrow 0} \sup _{t \leq T}\left|\int_{0}^{t}\left(h\left(\dot{\bar{\varepsilon}}, \xi_{s / \varepsilon^{\alpha}}\right) \varphi, v^{\varepsilon}(s)\right) d s\right|=0
$$

for any test function $\varphi$.

Proof Denote by $H z$-periodic vector function $(z, y) \mapsto H(z, y)$ given by

$$
\operatorname{div}_{z} H(z, y)=h(z, y)-\langle h(\cdot, y)\rangle, \quad|H| \leq c(1+|y|)^{\theta} ;
$$

for instance, one can put $H(z, y)=\nabla_{z} \hat{H}(z, y)$ where $z$-periodic $\hat{H}(z, y)$ solves the equation $\Delta_{z} \hat{H}(z, y)=h(z, y)-\langle h(\cdot, y)\rangle$. In view of the identity:

$$
\begin{aligned}
\int_{0}^{t}\left(h\left(\dot{\bar{\varepsilon}}, \xi_{s / \varepsilon^{\alpha}}\right) \varphi, v^{\varepsilon}(s)\right) d s= & \varepsilon \int_{0}^{t}\left(H\left(\dot{\bar{\varepsilon}}, \xi_{s / \varepsilon^{\alpha}}\right), \nabla\left(\varphi v^{\varepsilon}(s)\right)\right) d s \\
& +\int_{0}^{t}\left\langle h\left(\cdot, \xi_{s / \varepsilon^{\alpha}}\right)\right\rangle\left(\varphi, v^{\varepsilon}(s)\right) d s
\end{aligned}
$$

and the estimates

$$
\begin{aligned}
& E \sup _{t \leq T}\left|\int_{0}^{t} \sum_{i}\left(H^{i}\left(\dot{\bar{\varepsilon}}_{\bar{\varepsilon}}, \xi_{s / \varepsilon^{\alpha}}\right),\left(\varphi v^{\varepsilon}(s)\right)_{x_{i}}\right) d s\right| \\
& \leq C E\left(\left\|v^{\varepsilon}(t)\right\|_{L^{2}\left((0, T) ; H^{1}\left(\mathbb{R}^{n}\right)\right)}^{2}\right)^{1 / 2} \leq C_{1}
\end{aligned}
$$

it is sufficient to obtain the following limit relation:

$$
P-\lim _{\varepsilon \downarrow 0} \sup _{t \leq T}\left|\int_{0}^{t}\left\langle h\left(\cdot, \xi_{s / \varepsilon^{\alpha}}\right)\right\rangle\left(\varphi, v^{\varepsilon}(s)\right) d s\right|=0 .
$$

Note that the family of distributions of the processes $\left(\varphi, v^{\varepsilon}\right)$ is relatively compact in $\mathcal{C}[0, T]$. That is, for any $\gamma>0$, there exist $N>0$ and $f^{1}, \ldots, f^{N} \in$ $\mathcal{C}\left([0, T] ; \mathbb{R}^{n}\right)$ such that: $P\left(\mathcal{A}_{\gamma}\right)<\gamma$ for all $\varepsilon>0$ with

$$
\mathcal{A}_{\gamma}=\bigcap_{k=1}^{N}\left\{\sup _{0 \leq t \leq T}\left|\left(\varphi, v^{\varepsilon}(t)\right)-f^{k}(t)\right|>\gamma\right\} .
$$


Hence $\mathcal{A}_{\gamma}^{c}$ is of the form $\cup_{k} \mathcal{B}_{\gamma}^{k}, \mathcal{B}_{\gamma}^{k}=\left\{\sup _{0 \leq t \leq T}\left|\left(\varphi, u^{\varepsilon}(t)\right)-f^{k}(t)\right| \leq \gamma\right\}$, so $\mathcal{A}_{\gamma}^{c}=\cup_{k} \tilde{\mathcal{B}}_{\gamma}^{k}$, where $\tilde{\mathcal{B}}_{\gamma}^{k} \subset \mathcal{B}_{\gamma}^{k}$ and $\tilde{\mathcal{B}}_{\gamma}^{k} \cap \tilde{\mathcal{B}}_{\gamma}^{l}=\emptyset$ for $k \neq l$. Then,

$$
\begin{aligned}
& E \sup _{t \leq T}\left|\int_{0}^{t}\left\langle h\left(\cdot, \xi_{s / \varepsilon^{\alpha}}\right)\right\rangle\left(\varphi, v^{\varepsilon}\right) d s\right| \\
&=E {\left[\mathbf{1}_{\mathcal{A}_{\gamma}} \sup _{t \leq T}\left|\int_{0}^{t}\left\langle h\left(\cdot, \xi_{s / \varepsilon^{\alpha}}\right)\right\rangle\left(\varphi, v^{\varepsilon}\right) d s\right|\right] } \\
& \quad+E\left[\mathbf{1}_{\mathcal{A}_{\gamma}^{c}} \sup _{t \leq T}\left|\int_{0}^{t}\left\langle h\left(\cdot, \xi_{s / \varepsilon^{\alpha}}\right)\right\rangle\left(\varphi, v^{\varepsilon}\right) d s\right|\right] \\
& \leq C \sqrt{\gamma}+\sum_{k} E\left[\mathbf{1}_{\tilde{B}_{\gamma}^{k}} \sup _{t \leq T}\left|\int_{0}^{t}\left\langle h\left(\cdot, \xi_{s / \varepsilon^{\alpha}}\right)\right\rangle\left(\varphi, v^{\varepsilon}\right) d s\right|\right] \\
& \leq C_{1} \sqrt{\gamma}+\sum_{k} E\left[\sup _{t \leq T}\left|\int_{0}^{t}\left\langle h\left(\cdot, \xi_{s / \varepsilon^{\alpha}}\right)\right\rangle f^{k}(s) d s\right|\right]
\end{aligned}
$$

and the required statement follows from the averaging principle (Theorem 9.6.1 of [10]) if we pass to the limit in the right hand side first as $\varepsilon \downarrow 0$ and then as $\gamma \downarrow 0$.

To finish the proof, let us introduce a bounded continuous functional $\Phi_{\varphi}(u)$ on the space $\Omega_{T}$ :

$$
\begin{aligned}
\Phi_{\varphi}(u)=1 \wedge \sup _{t \leq T} \mid(\varphi(t), u(t)) & -\left(\varphi(0), u_{0}\right)-\int_{0}^{t}\left(\left(\varphi_{s}(s), u(s)\right) d s\right. \\
& -\int_{0}^{t}\left(\sum_{i j} \hat{a}^{i j} \varphi_{x_{i} x_{j}}(s), u(s)\right) d s \mid
\end{aligned}
$$

From (29), we get $\lim _{\varepsilon \downarrow 0} E \Phi_{\varphi}\left(v^{\varepsilon}\right)=0$, so for any limiting point $Q$ of the family $\tilde{Q}^{\varepsilon}$, we obtain $E^{Q} \Phi_{\varphi}(u)=0$ and therefore any limiting measure $Q$ is concentrated on the weak solution of the deterministic equation (28). Thus, the uniqueness of the solution of the latter problem implies the desired convergence in probability.

Case $\alpha=2$

We follow the same scheme as above: let $\hat{v}(x, t)$ be the solution of the Cauchy problem

$$
\hat{v}_{t}=\operatorname{div}(\hat{a} \nabla \hat{v})-\hat{b} \nabla \hat{v}+\hat{\tilde{c}} \hat{v}, \hat{v}(x, 0)=u_{0}(x),
$$

$\mathrm{RR} \mathrm{n}^{\circ} 3520$ 
where $\hat{a}$ and $\hat{b}$ were defined in Section 3.1, and $\hat{\tilde{c}}=\overline{\langle\tilde{G} \tilde{c}\rangle}$.

We are going to prove that any limit point of the family $\left\{\tilde{Q}^{\varepsilon}\right\}$ is $\delta$-type measure concentrated on $\hat{v}$.

Proposition 4.4 For any test function $\varphi(t, x) \in C^{\infty}\left([0, T] ; C_{0}^{\infty}\left(R^{n}\right)\right)$ the following limit relation holds:

$$
\begin{aligned}
\lim _{\varepsilon \downarrow 0} E \sup _{t \leq T} & \mid\left(\varphi(t), v^{\varepsilon}(t)\right)-\left(\varphi(0), u_{0}\right)-\int_{0}^{t}\left(\varphi_{s}(s), v^{\varepsilon}(s)\right) d s \\
- & \int_{0}^{t}\left(\left[\sum_{i j} \hat{a}^{i j} \varphi_{x_{i} x_{j}}(s)+\hat{b} \nabla \varphi(s)+\hat{\tilde{c}} \varphi(s)\right], v^{\varepsilon}(s)\right) d s \mid=0 .
\end{aligned}
$$

Proof Consider the auxiliary process:

$$
H^{\varepsilon}(t)=\left(\varphi, v^{\varepsilon}(t)\right)+\varepsilon \sum_{k}\left(\Psi^{k}\left(\dot{\bar{\varepsilon}}, \xi_{t / \varepsilon^{2}}\right) \varphi_{x_{k}}, v^{\varepsilon}(t)\right)+\varepsilon\left(\tilde{G}\left(\dot{\bar{\varepsilon}}, \xi_{t / \varepsilon^{2}}\right) \varphi, v^{\varepsilon}(t)\right)
$$

where $\Psi^{k}$ is defined by (13) and $\tilde{G}(z, y)$ satisfies the equation

$$
(\mathcal{A}+\mathcal{L}) \tilde{G}(z, y)=-\tilde{c}(z, y) .
$$

It follows from Proposition 4.1, (E1)-(E2), and Proposition 2.6 that

$$
\lim _{\varepsilon \downarrow 0} E \sup _{t \leq T}\left|H^{\varepsilon}(t)-\left(\varphi, v^{\varepsilon}(t)\right)\right|=0 .
$$

Applying Ito's formula to $H^{\varepsilon}(t)$ gives:

$$
\begin{aligned}
d H^{\varepsilon}=\sum_{i j}\left[\left(a^{\varepsilon, i j} \varphi_{x_{i} x_{j}}, v^{\varepsilon}\right)+\varepsilon^{-1}\left(a_{z_{i}}^{\varepsilon, i j} \varphi_{x_{j}}, v^{\varepsilon}\right)\right] d t+\varepsilon^{-1}\left(\tilde{c}^{\varepsilon} \varphi, v^{\varepsilon}\right) d t \\
+\left(\nabla_{y} \tilde{G}^{\varepsilon} \varphi+\sum_{k} \nabla_{y} \Psi^{k} \varphi_{x_{k}}, v^{\varepsilon}\right) \cdot \sigma^{\varepsilon} d W_{t} \\
+\left[\varepsilon^{-1} \sum_{k}\left(\mathcal{L} \Psi^{k} \varphi_{x_{k}}, v^{\varepsilon}\right)+\sum_{k}\left(\Psi^{k} \tilde{c}^{\varepsilon} \varphi_{x_{k}}, v^{\varepsilon}\right)\right. \\
\quad+\varepsilon^{-1} \sum_{k}\left(\mathcal{A} \Psi^{k} \varphi_{x_{k}}, v^{\varepsilon}\right) \\
\quad+\sum_{i j k}\left(\left(a^{\varepsilon, i j} \Psi^{k}\right)_{z_{i}} \varphi_{x_{k} x_{j}}+a^{\varepsilon, i j} \Psi_{z_{j}}^{k} \varphi_{x_{k} x_{i}}, v^{\varepsilon}\right) \\
\quad+\varepsilon \sum_{i j k}\left(a^{\varepsilon, i j} \Psi^{k} \varphi_{x_{i} x_{j} x_{k}}, v^{\varepsilon}\right) \\
\quad+\varepsilon^{-1}\left(\mathcal{L} \tilde{G}^{\varepsilon} \varphi, v^{\varepsilon}\right)+\left(\tilde{G}^{\varepsilon} \tilde{c}^{\varepsilon} \varphi, v^{\varepsilon}\right) \\
\quad+\varepsilon^{-1}\left(\mathcal{A} \tilde{G}^{\varepsilon} \varphi, v^{\varepsilon}\right)+\sum_{i j}\left(\left(a^{\varepsilon, i j} \tilde{G}^{\varepsilon}\right)_{z_{i}} \varphi_{x_{j}}+a^{\varepsilon, i j} \tilde{G}_{z_{j}}^{\varepsilon} \varphi_{x_{i}}, v^{\varepsilon}\right) \\
\left.\quad+\varepsilon \sum_{i j}\left(a^{\varepsilon, i j} \tilde{G}^{\varepsilon} \varphi_{x_{i} x_{j}}, v^{\varepsilon}\right)\right] d t .
\end{aligned}
$$


Taking into account Equations (35)-(13) we simplify the expression in the right hand side as follows:

$$
\begin{gathered}
d H^{\varepsilon}=\left[\sum_{i j}\left(a^{\varepsilon, i j} \varphi_{x_{i} x_{j}}, v^{\varepsilon}\right)+\sum_{i j k}\left(\left(a^{\varepsilon, i j} \Psi^{k}\right)_{z_{i}} \varphi_{x_{k} x_{j}}+a^{\varepsilon, i j} \Psi_{z_{j}}^{k} \varphi_{x_{k} x_{i}}, v^{\varepsilon}\right)\right. \\
\quad+\sum_{k}\left(\Psi^{k} \tilde{c}^{\varepsilon} \varphi_{x_{k}}, v^{\varepsilon}\right)+\left(\tilde{G}^{\varepsilon} \tilde{c}^{\varepsilon} \varphi, v^{\varepsilon}\right) \\
\left.+\sum_{i j}\left(\left(a^{\varepsilon, i j} \tilde{G}^{\varepsilon}\right)_{z_{i}} \varphi_{x_{j}}+a^{\varepsilon, i j} \tilde{G}_{z_{j}}^{\varepsilon} \varphi_{x_{i}}, v^{\varepsilon}\right)+\left(\varphi_{t}, v^{\varepsilon}\right)\right] d t \\
+\left(\nabla_{y} \tilde{G}^{\varepsilon} \varphi+\sum_{k} \nabla_{y} \Psi^{k} \varphi_{x_{k}}, v^{\varepsilon}\right) \cdot \sigma^{\varepsilon} d W_{t}+\varepsilon d R^{\varepsilon}(t)
\end{gathered}
$$

where $E \sup _{t \leq T}\left|R^{\varepsilon}(t)\right| \leq C$.

Denote by $M^{\varepsilon}(t)$ the stochastic term on the right hand side of the latter formula:

$$
M^{\varepsilon}(t)=\int_{0}^{t}\left(\nabla_{y} \tilde{G}\left(\dot{\bar{\varepsilon}}, \xi_{s / \varepsilon^{2}}\right) \varphi+\sum_{k} \nabla_{y} \Psi^{k} \varphi_{x_{k}}, v^{\varepsilon}(s)\right) \cdot \sigma\left(\xi_{s / \varepsilon^{2}}\right) \varepsilon d W_{s / \varepsilon^{2}},
$$

clearly $M^{\varepsilon}(t)$ is $\mathcal{F}_{t}^{\varepsilon}$-adapted square integrable martingale.

Proposition 4.5 The following limit relation holds true:

$$
\lim _{\varepsilon \rightarrow 0} E \sup _{t \leq T}\left|M^{\varepsilon}(t)\right|=0 \text {. }
$$

Proof It is easy to see that the operator $\mathcal{L}$ does commute with averaging in variable $z$. Thus, taking the mean value in $z$ in Equations (13) and (35), we find $\left\langle\nabla_{y} \Psi(\cdot, y)\right\rangle=0$ and $\left\langle\nabla_{y} \tilde{G}(\cdot, y)\right\rangle=0$ for all $y$. Therefore,

$$
\begin{gathered}
E \sup _{t \leq T}\left|\int_{0}^{t}\left[\sum_{k}\left(\nabla_{y} \Psi^{k} \varphi_{x_{k}}, v^{\varepsilon}(s)\right)+\left(\nabla_{y} \tilde{G}, \varphi v^{\varepsilon}(s)\right)\right] \cdot \sigma\left(\xi_{s / \varepsilon^{2}}\right) d W_{s}\right| \\
=\varepsilon E \sup _{t \leq T} \mid \int_{0}^{t}\left[\sum_{k}\left(H^{k}, \nabla\left(\varphi_{x_{k}} v^{\varepsilon}(s)\right)\right)+\left(\tilde{H}, \nabla\left(\varphi v^{\varepsilon}(s)\right)\right)\right] \\
\cdot \sigma\left(\xi_{s / \varepsilon^{2}}\right) d W_{s} \mid
\end{gathered}
$$

where $H^{k}(z, y)$ and $\tilde{H}(z, y)$ are given by

$$
\begin{aligned}
\operatorname{div}_{z} H^{k}(z, y) & =\nabla_{y} \Psi^{k}(z, y) \\
\operatorname{div}_{z} \tilde{H}(z, y) & =\nabla_{y} \tilde{G}(z, y) .
\end{aligned}
$$

$\mathrm{RR} \mathrm{n}^{\circ} 3520$ 
By virtue of (E1)-(E2), and the Burkholder-Davis-Gundy inequality we have

$$
\begin{aligned}
\lim _{\varepsilon \downarrow 0} E \sup _{t \leq T}\left|\sum_{k} \int_{0}^{t}\left(\nabla_{y} \Psi^{k} \varphi_{x_{k}}, v^{\varepsilon}(s)\right) \cdot \sigma\left(\xi_{s / \varepsilon^{2}}\right) d W_{s}\right| & =0, \\
\lim _{\varepsilon \downarrow 0} E \sup _{t \leq T}\left|\int_{0}^{t}\left(\nabla_{y} \tilde{G} \varphi, v^{\varepsilon}(s)\right) \cdot \sigma\left(\xi_{s / \varepsilon^{2}}\right) d W_{s}\right| & =0 .
\end{aligned}
$$

Passing to the limit in the right hand side of (36) by means of Propositions 4.5 and 4.3, we complete the proof of Proposition 4.4.

Now, the convergence of $v^{\varepsilon}$ in probability can be derived in the same way as in the case $\alpha<2$.

Case $\alpha>2$

The approach used in this case is quite similar to that of the preceding cases so we consider it briefly. We introduce a function $\hat{v}(x, t)$ to satisfy the Cauchy problem

$$
\hat{v}_{t}=\operatorname{div}(\hat{a} \nabla \hat{v})+\hat{c} \hat{v}, \hat{v}(x, 0)=u_{0}(x),
$$

with $\hat{a}$ and $\hat{c}$ defined in Section 3.3. The proof of the fact that $v^{\varepsilon}$ converges to $\hat{v}$ in probability relies on the following

Proposition 4.6 For any test function $\varphi(x, t) \in C^{\infty}\left([0, T] ; C_{0}^{\infty}\left(R^{n}\right)\right)$ the following limit relation holds

$$
\begin{array}{r}
\lim _{\varepsilon \downarrow 0} E \sup _{t \leq T} \mid \\
\mid\left(\varphi(t), v^{\varepsilon}(t)\right)-\left(\varphi(0), u_{0}\right)-\int_{0}^{t}\left(\varphi_{s}(s), v^{\varepsilon}(s)\right) d s \\
-\int_{0}^{t}\left(\sum_{i j} \hat{a}^{i j} \varphi_{x_{i} x_{j}}(s)+\hat{c} \varphi(s), v^{\varepsilon}(s)\right) d s \mid=0
\end{array}
$$

Proof We define the functions $G^{l} \in \bar{H}^{1}\left(\mathbb{T}^{n}\right), g^{l} \in \bar{H}^{1}\left(\mathbb{T}^{n} \times \mathbb{R}^{d}\right), l=0, \ldots, K$, to be solutions of the system of equations:

$$
\left\{\begin{aligned}
\overline{\mathcal{A}} G^{0}(z) & =-\overline{c(z, \cdot)} \\
\mathcal{L} g^{0}(z, y) & =-\left[\tilde{c}(z, y)-\overline{c(z, \cdot)}+\mathcal{A} G^{0}(z)-\overline{\mathcal{A}} G^{0}(z)\right] \\
\overline{\mathcal{A}} G^{l}(z) & =-\overline{\mathcal{A} g^{l-1}(z, \cdot)} \\
\mathcal{L} g^{l}(z, y) & =-\left[(\mathcal{A}-\overline{\mathcal{A}}) G^{l}(z)+\mathcal{A} g^{l-1}(z, y)-\overline{\mathcal{A} g^{l-1}(z, \cdot)}\right]
\end{aligned}\right.
$$


and the functions $\Psi^{k l} \in \bar{H}^{1}\left(\mathbb{T}^{n}\right)$ and $\Phi^{k l} \in \bar{H}_{\rho}^{1}\left(\mathbb{T}^{n} \times \mathbb{R}^{d}\right), k=1, \ldots, n$, to satisfy the system

$$
\begin{aligned}
& \left\{\begin{aligned}
\overline{\mathcal{A}} \Psi^{k 0}(z) & =-\sum_{i} \overline{a_{z_{i}}^{i k}(z, \cdot)} \\
\mathcal{L} \Phi^{k 0}(z, y) & =-\left[(\mathcal{A}-\overline{\mathcal{A}}) \Psi^{k 0}(z)+\sum_{i}\left(a_{z_{i}}^{i k}(z, y)-\overline{a_{z_{i}}^{i k}(z, \cdot)}\right)\right]
\end{aligned}\right. \\
& \left\{\begin{aligned}
\overline{\mathcal{A}} \Psi^{k l}(z) & =-\overline{\mathcal{A} \Phi^{k(l-1)}(z, \cdot)} \\
\mathcal{L} \Phi^{k l}(z, y) & =-\left[(\mathcal{A}-\overline{\mathcal{A}}) \Psi^{k l}(z)+\mathcal{A} \Phi^{k(l-1)}(z, y)-\overline{\mathcal{A} \Phi^{k(l-1)}(z, \cdot)}\right]
\end{aligned}\right.
\end{aligned}
$$

$k=1, \ldots, n, l=0, \ldots, K$ with $K=[1 / \delta]+1$, and $\delta=\alpha-2>0$.

Applying Ito's formula to the expression

$$
\begin{aligned}
\left(\varphi(t), v^{\varepsilon}(t)\right) & +\sum_{l} \varepsilon^{1+l \delta} \sum_{k}\left(\Psi^{k l}(\dot{\bar{\varepsilon}}) \varphi_{x_{k}}(t), v^{\varepsilon}(t)\right) \\
& +\sum_{l} \varepsilon^{1+(l+1) \delta} \sum_{k}\left(\Phi^{k l}\left(\dot{\bar{\varepsilon}}, \xi_{t / \varepsilon^{\alpha}}\right) \varphi_{x_{k}}(t), v^{\varepsilon}(t)\right) \\
& +\sum_{l} \varepsilon^{1+l \delta}\left(G^{l}(\dot{\dot{\varepsilon}}) \varphi(t), v^{\varepsilon}(t)\right) \\
& +\sum_{l} \varepsilon^{1+(l+1) \delta}\left(g^{l}\left(\dot{\bar{\varepsilon}}, \xi_{t / \varepsilon^{\alpha}}\right) \varphi(t), v^{\varepsilon}(t)\right) .
\end{aligned}
$$

we obtain after simple transformations

$$
\begin{aligned}
\left(\varphi(t), v^{\varepsilon}(t)\right)= & \left(\varphi(0), u_{0}\right) \\
& +\sum_{i j} \int_{0}^{t}\left(\varphi_{s}(s) v^{\varepsilon}(s)\right) d s+\sum_{i j} \int_{0}^{t}\left(a^{\varepsilon, i j} \varphi_{x_{i} x_{j}}(s), v^{\varepsilon}(s)\right) d s \\
& +\sum_{k} \int_{0}^{t}\left(\Psi^{k 0} \varphi_{x_{k}}(s) \tilde{c}^{\varepsilon}, v^{\varepsilon}(s)\right) d s \\
& +\sum_{k} \sum_{i j} \int_{0}^{t}\left(\left(a^{\varepsilon, i j} \Psi^{k 0}\right)_{z_{i}} \varphi_{x_{k} x_{j}}(s)\right. \\
& \left.\quad+a^{\varepsilon, i j} \Psi_{z_{j}}^{k 0} \varphi_{x_{k} x_{i}}(s), v^{\varepsilon}(s)\right) d s \\
& +\sum_{i j} \int_{0}^{t}\left(\left(a^{\varepsilon, i j} G^{0}\right)_{z_{i}} \varphi_{x_{j}}(s)+a^{\varepsilon, i j} G_{z_{j}}^{0} \varphi_{x_{i}}(s), v^{\varepsilon}(s)\right) d s \\
& +\int_{0}^{t}\left(G^{0} \tilde{c}^{\varepsilon} \varphi(s), v^{\varepsilon}(s)\right) d s+\varepsilon^{\delta / 2} \tilde{R}^{\varepsilon}(t)
\end{aligned}
$$

where $E \sup _{t<T}\left|\tilde{R}^{\varepsilon}(t)\right| \leq C$.

Therefore, $\left(\varphi(t), v^{\varepsilon}(t)\right)$ can be represented as:

$$
\begin{aligned}
\left(\varphi(t), v^{\varepsilon}(t)\right)= & \left(\varphi(0), u_{0}\right)+\int_{0}^{t}\left(\varphi_{s}(s), v^{\varepsilon}(s)\right) d s \\
& +\sum_{m} \int_{0}^{t}\left(h^{m}\left(\dot{\bar{\varepsilon}}, \xi_{\frac{s}{\varepsilon^{\alpha}}}\right) \tilde{\varphi}^{m}(s), v^{\varepsilon}(s)\right) d s+\varepsilon^{\delta / 2} \tilde{R}^{\varepsilon}(t)
\end{aligned}
$$

$\mathrm{RR} \mathrm{n}^{\circ} 3520$ 
where $\tilde{\varphi}^{m}$ denotes one of the functions $\varphi, \varphi_{x_{i}}, \varphi_{x_{i} x_{j}}$, and $h^{m}$ one of the functions $a^{i j}, \Psi^{k 0}, \Psi_{x_{i}}^{k 0}, G^{0}, G_{x_{j}}^{0}, \tilde{c}$.

Hence,

$$
\begin{aligned}
& \left(\varphi(t), v^{\varepsilon}(t)\right)-\left(\varphi(0), u_{0}\right)-\int_{0}^{t}\left(\varphi_{s}(s), v^{\varepsilon}(s)\right) d s \\
& \quad-\sum_{m} \int_{0}^{t} \overline{\left\langle h^{m}\right\rangle}\left(\tilde{\varphi}^{m}(s), v^{\varepsilon}(s)\right) d s \\
& \quad=\varepsilon^{\delta / 2} R^{\varepsilon}(t)+\sum_{m} \int_{0}^{t}\left(\left[h^{m}\left(\dot{\bar{\varepsilon}}, \xi_{\frac{s}{\varepsilon^{\alpha}}}\right)-\overline{\left\langle h^{m}\right\rangle}\right] \tilde{\varphi}^{m}(s), v^{\varepsilon}(s)\right) d s
\end{aligned}
$$

and to complete the proof it is sufficient to show that

$$
P-\lim _{\varepsilon \downarrow 0} \sup _{t \leq T}\left|\int_{0}^{t}\left(\left[h^{m}\left(\dot{\bar{\varepsilon}}, \xi_{\frac{s}{\varepsilon^{\alpha}}}\right)-\overline{\left\langle h^{m}\right\rangle} \tilde{\varphi}^{m}(s)\right], v^{\varepsilon}(s)\right) d s\right|=0
$$

for any test function $\varphi(t, x)$.

This relation is a direct consequence of Proposition 4.3.

It remains to show that the first order terms in the limit equation are equal to zero or, equivalently, that

$$
\left\langle\bar{c} \Psi^{k 0}+\bar{a} \nabla G^{0}\right\rangle=0 .
$$

Indeed, Definitions (39) of $G^{0}$ and (40) of $\Psi^{k 0}$ lead to:

$$
\begin{aligned}
\left\langle\bar{c} \Psi^{k 0}+\bar{a} \nabla G^{0}\right\rangle & =\left\langle-\left(\overline{\mathcal{A}} G^{0}\right) \Psi^{k 0}+\bar{a} \nabla G^{0}\right\rangle \\
& =\left\langle G^{0}\left(\overline{\mathcal{A}} \Psi^{k 0}+\nabla \bar{a}\right)\right\rangle \\
& =0 .
\end{aligned}
$$

\subsection{Convergence in law of the solution of initial problem - Description of the limit distribution}

In this section we establish the convergence of the family $\left\{u^{\varepsilon}\right\}$ in law. We start by studying the limit distribution of the exponent from (24). According to [13], Theorem 3, the family of integrals

$$
J_{t}^{\varepsilon, \alpha}=\frac{1}{\varepsilon^{\alpha / 2}} \int_{0}^{t}\left\langle c\left(\cdot, \xi_{s / \varepsilon^{\alpha}}\right)\right\rangle d s
$$


converges in law in $\mathcal{C}[0, T]$ as $\varepsilon \rightarrow 0$, to $\lambda \hat{W}_{t}$, where $\hat{W}_{t}$ is a standard onedimensional Brownian motion and $\lambda$ is defined as follows:

$$
\lambda^{2}=2 \overline{q \nabla G \cdot \nabla G}, \mathcal{L} G=-\langle c(\cdot, y)\rangle .
$$

In fact, this definition coincides with that of Sections 3.1, 3.2.

In particular, the above result implies that for $\alpha>2$

$$
P-\lim _{\varepsilon \rightarrow 0} \frac{1}{\varepsilon} \int_{0}^{t}\left\langle c\left(\cdot, \xi_{s / \varepsilon^{\alpha}}\right)\right\rangle d s=0
$$

and, therefore,

$$
P-\lim _{\varepsilon \rightarrow 0} \exp \left(\frac{1}{\varepsilon} \int_{0}^{t}\left\langle c\left(\cdot, \xi_{s / \varepsilon^{\alpha}}\right)\right\rangle d s\right)=1 .
$$

For $\alpha \leq 2$ the family $\exp \left(J_{t}^{\varepsilon, \alpha}\right)$ converges in law to the distribution of $\exp \left(\lambda \hat{W}_{t}\right)$.

In order to pass to the limit in the product $\exp \left(J_{t}^{\varepsilon, \alpha}\right) v^{\varepsilon}$, we represent it in the form:

$$
\exp \left(J_{t}^{\varepsilon, \alpha}\right) v^{\varepsilon}=\exp \left(J_{t}^{\varepsilon, \alpha}\right) \hat{v}+\exp \left(J_{t}^{\varepsilon, \alpha}\right)\left(v^{\varepsilon}-\hat{v}\right)
$$

It is easy to see that the map $\nu(\cdot) \mapsto \nu(\cdot) \hat{v}$ is a continuous map from $\mathcal{C}[0, T]$ to $\Omega_{T}$. Thus, the first term on the right hand side of (43) converges in law to $\exp \left(\lambda \hat{W}_{t}\right) \hat{v}$ in $\Omega_{T}$ as $\varepsilon \rightarrow 0$.

The second term converges in probability to zero. Indeed, by Prokhorov's theorem [2], for any $\gamma>0$ there is a compact subset $K^{\gamma}$ of $\mathcal{C}[0, T]$ such that

$$
P\left\{\exp \left(J^{\varepsilon, \alpha}\right) \in K^{\gamma}\right\} \geq 1-\gamma .
$$

For a finite $\gamma$-net $f_{1}(t), f_{2}(t), \ldots, f_{N}(t)$ in $K^{\gamma}$, we construct mutually nonintersecting sets $K_{1}^{\gamma}, K_{2}^{\gamma}, \ldots, K_{N}^{\gamma}$ such that $K_{l}^{\gamma}$ belongs to $\gamma$-neighborhood of $f_{l}(\cdot), l=1,2, \ldots, N$, and $K^{\gamma}=\bigcup_{l} K_{l}^{\gamma}$. Now one can rewrite the expression studied as follows:

$$
\begin{aligned}
\exp \left(J_{t}^{\varepsilon, \alpha}\right)\left(v^{\varepsilon}-\hat{v}\right)= & \mathbf{1}_{\exp \left(J_{t}^{\varepsilon, \alpha}\right) \notin K^{\gamma}} \exp \left(J_{t}^{\varepsilon, \alpha}\right)\left(v^{\varepsilon}-\hat{v}\right) \\
& +\sum_{l} \mathbf{1}_{\exp \left(J_{t}^{\varepsilon, \alpha}\right) \in K_{l}^{\gamma}} f_{l}(t)\left(v^{\varepsilon}-\hat{v}\right) \\
& +\sum_{l} \mathbf{1}_{\exp \left(J_{t}^{\varepsilon, \alpha}\right) \in K_{l}^{\gamma}}\left[\exp \left(J_{t}^{\varepsilon, \alpha}\right)-f_{l}(t)\right]\left(v^{\varepsilon}-\hat{v}\right)
\end{aligned}
$$

$\mathrm{RR} \mathrm{n}^{\circ} 3520$ 
It remains to notice that by (E1) and (E2)

$$
\begin{aligned}
& \left\|\mathbf{1}_{\exp \left(J_{t}^{\varepsilon, \alpha}\right) \in K_{l}^{\gamma}}\left[\exp \left(J_{t}^{\varepsilon, \alpha}\right)-f_{l}(t)\right]\left(v^{\varepsilon}-\hat{v}\right)\right\|_{L^{\infty}\left(0, T ; L^{2}\left(R^{n}\right)\right)} \\
& \quad+\left\|\mathbf{1}_{\exp \left(J_{t}^{\varepsilon, \alpha}\right) \in K_{l}^{\gamma}}\left[\exp \left(J_{t}^{\varepsilon, \alpha}\right)-f_{l}(t)\right]\left(v^{\varepsilon}-\hat{v}\right)\right\|_{L^{2}\left(0, T ; H^{1}\left(R^{n}\right)\right)} \leq c \gamma,
\end{aligned}
$$

and pass to the limit in (44) first as $\varepsilon \rightarrow 0$ and then as $\gamma \rightarrow 0$.

Finally, in order to show that the limit distributions obtained satisfy the limit SPDEs (8) or (11) one can apply Ito's formula to the product $\exp \left(\lambda \hat{W}_{t}\right) \hat{v}(t, x)$ and use the auxiliary homogenized equations (28) or (34) respectively.

With evident simplifications, one can use (42) to pass to the limit in the product:

$$
u^{\varepsilon}(t, x)=\exp \left(\frac{1}{\varepsilon} \int_{0}^{t}\left\langle c\left(\cdot, \xi_{s / \varepsilon^{\alpha}}\right)\right\rangle d s\right) v^{\varepsilon}(t, x)
$$

in the case $\alpha>2$, and to show that the functions $v^{\varepsilon}$ converge in probability in $\Omega_{T}$ to the solution $\hat{v}$ of problem (37). 


\section{References}

[1] M. AVELLANEDA and A.J. MAJDA. Simple examples with features of renormalization for turbulent transport. Phil. Trans. R. Soc. Lond. A, 346:205-233, 1994.

[2] P. BILLINGSLEY. Convergence of Probability Measures. John Wiley \& Sons, New York, 1968.

[3] R. BOUC and É. PARDOUX. Asymptotic analysis of PDEs with whideband noise disturbances, and expansion of the moments. Stochastic Analysis and Applications, 2(4):369-422, 1984.

[4] A. BOURGEAT, A. MIKELIC, and S. WRIGHT. Stochastic two-scale convergence in the mean and applications. Journal für die Reine ande Angewandte Mathematik, 456:19-51, 1994.

[5] A. FANNJIANG and G. PAPANICOLAOU. Convection enhanced diffusion for random flows. Journal of Statistical Physics, 88:1033-1076, 1997.

[6] U. HORNUNG, editor. Homogenization and Porous Media, volume 6 of IAM. Springer Verlag, 1997.

[7] V. V. JIKOV, S. M. KOZLOV, and O. A. OLEINIK. Homogenization of Differential Operators and Integral Functionals. Springer Verlag, 1994.

[8] M.L. KLEPTSYNA and A.L. PIATNITSKI. Homogenization of random parabolic operators. GAKUTO International Series, Mathemathical Sciences and Applications, 9:241-255, 1997.

[9] S.M. KOZLOV. Averaging of random operators. Math. USSR Sbornik, 37(2):167-180, 1980.

[10] R.Sh. LIPTSER and A.N. SHIRYAEV. Theory of Martingales. Mathematics and its Applications (Soviet Series). Kluwer Academic Publishers, Dordrecht, 1989.

[11] S. OLLA. Homogenization of diffusion processes in random fields. Technical report, Ecole Polytechnique, 1994.

$\mathrm{RR} \mathrm{n}^{\circ} 3520$ 
[12] G.C. PAPANICOLAOU and S.R.S VARADHAN. Diffusions with random coefficients. In G. Kallianpur, P.R. Krishnaiah, and J.K. Ghosh, editors, Statistics and Probability: An Essay in Honor of C.R. Rao, pages 547552. North-Holland, 1982.

[13] E. PARDOUX and A. Yu. VERETENNIKOV. On Poisson equation and diffusion approximation. part i. To appear.

[14] M. VIOT. Solutions Faibles d'Equations aux Dérivées Partielles Stochastiques Non Linéaires. Thèse, Université Paris VI, 1976. 


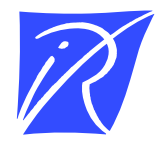

Unité de recherche INRIA Sophia Antipolis 2004, route des Lucioles - B.P. 93 - 06902 Sophia Antipolis Cedex (France)

Unité de recherche INRIA Lorraine : Technopôle de Nancy-Brabois - Campus scientifique 615, rue du Jardin Botanique - B.P. 101 - 54602 Villers lès Nancy Cedex (France)

Unité de recherche INRIA Rennes : IRISA, Campus universitaire de Beaulieu - 35042 Rennes Cedex (France)

Unité de recherche INRIA Rhône-Alpes : 655, avenue de l'Europe - 38330 Montbonnot St Martin (France)

Unité de recherche INRIA Rocquencourt : Domaine de Voluceau - Rocquencourt - B.P. 105 - 78153 Le Chesnay Cedex (France)

Éditeur

INRIA - Domaine de Voluceau - Rocquencourt, B.P. 105 - 78153 Le Chesnay Cedex (France)

http://www.inria.fr

ISSN 0249-6399 